\title{
Inhibition of miR-7 promotes angiogenesis in human umbilical vein endothelial cells by upregulating VEGF via KLF4
}

\author{
YI-ZE LI $^{1}$, LEI WEN ${ }^{1}$, XU WEI $^{2}$, QIAN-RONG WANG ${ }^{1}$, LONG-WEN XU ${ }^{1}$, \\ HONG-MEI ZHANG ${ }^{1}$ and WEN-CHAO LIU ${ }^{1}$ \\ ${ }^{1}$ Department of Clinical Oncology, Xijing Hospital, Fourth Military Medical University, Xi'an, Shaanxi 710032; \\ ${ }^{2}$ The Cadet Brigade, Fourth Military Medical University, Xi'an, Shaanxi 710032, P.R. China
}

Received February 18, 2016; Accepted March 26, 2016

DOI: $10.3892 /$ or.2016.4912

\begin{abstract}
Recent lentiviral-based microRNA (miRNA) library screening has identified miRNA-7 (miR-7) as an anti-angiogenic miRNA in human umbilical vein endothelial cells (HUVECs). However, the underlying mechanism of miR-7 in the suppression of angiogenesis remains largely unknown. In the present study, we report that miR-7 inhibition promoted angiogenesis by upregulating vascular endothelial growth factor (VEGF) and directly targeting Krüppel-like factor 4 (KLF4). Downregulation of miR-7 promoted tube formation of HUVECs, accompanied by upregulation of mRNA and protein levels of both VEGF and KLF4. miR-7 directly targeted KLF4 as demonstrated by luciferase reporter assay and miR-7 mimics decreased KLF4. Furthermore, bioinformatic analysis revealed the presence of multiple DNA-binding elements of KLF4 in the VEGF promoter. Chromatin immunoprecipitation (ChIP) demonstrated that the KLF4 antibody specifically pulled down the VEGF promoter in the HUVECs. Furthermore, ectopic overexpression of KLF4 induced VEGF mRNA and protein levels. In addition, KLF4 silencing inhibited the angiogenesis induced by the miR-7 inhibitor in the HUVECs. Our results demonstrated that KLF4 is a direct target of miR-7 and a transcription activator of VEGF. These findings indicate that the miR-7-KLF4-VEGF signaling axis plays an important role in the regulation of angiogenesis in HUVECs, suggesting that miR-7 is a potential agent for the development of anti-angiogenic therapeutics in vascular diseases and solid tumors.
\end{abstract}

Correspondence to: Professor Wen-Chao Liu or Professor Hong-Mei Zhang, Department of Clinical Oncology, Xijing Hospital, Fourth Military Medical University, 127 West Changle Road, Xi'an, Shaanxi 710032, P.R. China

E-mail: liuch@fmmu.edu.cn

E-mail: zhm@fmmu.edu.cn

Key words: HUVECs, UTR, microRNA, VEGF, RT-PCR, KLF4

\section{Introduction}

Normal physiology depends on an adequate blood supply. Angiogenesis is the formation of new vessels from pre-existing vessels. It is crucial to the development of embryos as well as several physiologic and pathologic processes including the female reproductive cycle, wound healing and the growth of solid tumors $(1,2)$. Endothelial cells (ECs) play an important role in angiogenesis and maintain vascular homeostasis (3). Compelling evidence has shown that vascular endothelial growth factor (VEGF) promotes endothelial cell proliferation and is deregulated in most vascular diseases and cancers $(4,5)$. However, the precise mechanism of the regulation of VEGF in human diseases particularly tumors remains not fully understood.

MicroRNAs (miRNAs) are highly conserved, singlestranded non-coding small RNAs, which regulate gene expression at the post-transcriptional level by inhibiting protein translation from mRNAs or by promoting degradation of mRNAs $(6,7)$. Numerous miRNAs are aberrantly expressed in cancer tissues and play a critical role in tumorigenesis and anticancer drug resistance $(8,9)$. miRNAs function as either tumor suppressors or oncogenes (oncomiRs). miRNA-7 (miR-7) was found to display tumor-suppressive function in colon cancer by targeting oncogenic YY1 (10), and in hepatocarcinoma via suppression of CCNE1 expression (11). Recently, a lentiviral-based miRNA library screening indicated that miR-7 is an anti-angiogenic miRNA, which was further validated in an animal model (12), suggesting that miR-7 is a novel anti-angiogenic therapeutic target. However, the underlying molecular mechanism of the suppression of angiogenesis by miR-7 is largely unknown.

Krüppel-like factors (KLFs) are a family of evolutionarily conserved zinc finger-containing transcription factors that bind 'CACCC' or 'GT-box' sites through a DNA-binding domain consisting of three zinc fingers positioned at their carboxyl-terminal end (13). Krüppel-like factor 4 (KLF4), Oct $3 / 4$, Sox 2 and c-Myc are the four defined critical factors inducing differentiated cells into pluripotent stem cells (14). Mounting evidence indicates that KLF4 is highly expressed in ECs and vascular smooth muscle cells (VSMCs) and acts as a critical regulator of vascular homeostasis (15). Diverse vasoprotective stimuli, including laminar shear stress and 
simvastatin, induce the expression of KLF4 in ECs $(16,17)$. Considering the overexpression of KLF4 in ECs and the anti-angiogenic function of miR-7, we hypothesized that downregulation of miR-7 may promote EC angiogenesis by targeting KLF4.

In the present study, we knocked down miR-7 by miR-7-specific antagomir in HUVECs and assessed angiogenesis. We found that miR-7 silencing resulted in enhanced angiogenesis, accompanied by upregulation of KLF4 and VEGF, revealing a novel angiogenesis-related miR-7-KLF4-VEGF signaling axis.

\section{Materials and methods}

Cell culture and transfection. HUVECs were obtained from the American Type Culture Collection (ATCC; Manassas, VA, USA) and cultured in Endothelial Cell Growth Medium-2 (EGM-2; Lonza, Portsmouth, NH, USA) at $37^{\circ} \mathrm{C}$ in a humidified atmosphere of $5 \% \mathrm{CO}_{2}$ as previously described (18). The HUVECs were transfected with oligonucleotides in 6-well plates using Lipofectamine 2000 reagent (Invitrogen, Carlsbad, CA, USA) according to the manufacturer's instructions. Briefly, 100 pmol of miR-7 mimics, miR-7 inhibitors (miR-ASO) or small interfering RNAs (siRNAs) against KLF4 (GenePharma, Shanghai, China) were formulated with $500 \mu \mathrm{l}$ serum-free RPMI-1640 medium (Invitrogen). The transfection complex was directly added to the cells and replaced with fresh complete medium $6 \mathrm{~h}$ later. Analyses of the effects of miRNAs or siRNAs on recipient cells were performed $48 \mathrm{~h}$ after transfection as previously described $(11,19)$.

Plasmid construction and luciferase report assay. The 3'-untranslated region (3'-UTR) of KLF4 was amplified from human genomic DNA and cloned into the pGL3-control vector (Promega, Madison, WI, USA) (19). Mutation of the miR-7 binding core sequence on the 3'-UTR region of the KLF4 gene was achieved using overlap extension PCR methods using primers as previously reported (19). HUVECs were seeded in 24-well plates and then transfected with $500 \mathrm{ng}$ of wildtype or mutant reporter plasmid, $25 \mathrm{ng}$ of Renilla luciferase expression vector, along with miR-7 mimics $(10 \mathrm{nM})$ or the negative control (10 nM) using Lipofectamine 2000 reagent. After $48 \mathrm{~h}$, the cell extract was used to determine firefly and Renilla luciferase activities with the Dual-Luciferase Reporter Assay system (Promega).

Quantitative real-time PCR. Total RNA was isolated from the cells using TRIzol reagent (Invitrogen) according to the manufacturer's instructions. RNAs were reverse transcribed into cDNA using miScript Reverse Transcription kit (Qiagen, Valencia, CA, USA). All PCR conditions were optimized to produce a single product in the exponential range. Quantitative real-time PCR was performed using SYBR-Green Master Mix and analyzed using an ABI Step One Real-Time PCR system (Applied Biosystems, Foster City, CA, USA). GAPDH was used as an internal loading control. Quantitative RT-PCR for KLF4, VEGF and GAPDH was performed using the following primers: KLF4 forward, 5'-CCCACATGAAGCGACTTCCC-3' and reverse 5'-CAGGTCCAGGAGATCGTTGAA-3'; VEGF forward, 5'-AGGGCAGAATCATCACGAAGT-3' and reverse 5'-AGGGTCTCGATTGGATGGCA-3'); GAPDH forward,
5'-TCACCAGGGCTGCTTTTAAC-3' and reverse, 5'-GACA AGCTTCCCGTTCTCAG-3'.

After placing the PCR tubes into the thermal cycler, the process entailed an initial denaturation step at $95^{\circ} \mathrm{C}$ for $10 \mathrm{~min}$ followed by a second step at $95^{\circ} \mathrm{C}$ for $15 \mathrm{sec}$ and $60^{\circ} \mathrm{C}$ for $30 \mathrm{sec}$ for 40 cycles with a melting curve analysis. The level of target mRNA was normalized to the level of GAPDH and was compared with the control. For the detection of miR-7, miRNA from cultured cells was extracted by mirVana miRNA Isolation kit (Ambion, Carlsbad, CA, USA) according to the manufacturer's instructions. TaqMan MicroRNA Reverse Transcription kit was used to produce cDNA for TaqMan MicroRNA assay (Applied Biosystems). Human miR-7 expression was quantified in real-time with FAM dye-labeled MGB-probes and normalized to snU6 (Applied Biosystems). Data were analyzed using the $\Delta \Delta \mathrm{C}_{\mathrm{T}}$ method.

Tube formation assay. HUVECs were seeded in a 6-well plate $\left(8 \times 10^{4}\right.$ cells/well) and transfected with $50 \mathrm{nM}$ miRNA mimics with Lipofectamine 2000 reagent in serum-free medium. After $4 \mathrm{~h}$, the medium was replaced with complete EGM-2 medium. A 96-well plate was coated with $50 \mu$ l Matrigel $^{\mathrm{TM}}$ (BD Biosciences, San Jose, CA, USA) and incubated for $30 \mathrm{~min}$ at $37^{\circ} \mathrm{C}$. After incubation for $48 \mathrm{~h}$, the transfected HUVECs were counted, prepared in a cell suspension in EGM-2 medium and seeded on top of the Matrigel ${ }^{\mathrm{TM}}$ (7,500 cells/well). After incubation for $17 \mathrm{~h}$, the wells were imaged with a Nikon TE2000 microscope at a magnification of x10. Tubule formation was quantified by counting the number of branch points in at least three independent wells under similar conditions using NIH ImageJ software.

ELISA detection of VEGF levels. The cell growth-conditioned medium from the above experiments was collected and analyzed for VEGF levels with immunosorbent assay (ELISA) kits (ACEL, Kanagawa, Japan) according to the manufacturer's instructions. The data are presented as mean \pm SEM.

Chromatin immunoprecipitation assay (ChIP). ChIP was performed as previously described (20). Briefly, HUVECs were grown and then washed twice with phosphate-buffered saline (PBS) and cross-linked using 1\% formaldehyde for $10 \mathrm{~min}$. Following lysis of the cells and sonication, DNA-protein complexes in the lysates were subjected to immunoprecipitation using anti-KLF4 (HPA002926; Sigma, St. Louis, MO, USA) or control normal IgG. After precipitation of the immunocomplex with protein G-agarose, isolated DNA was used as a template in PCR with specific primers spanning the target region of the VEGF promoter. Primers used for amplification of the human VEGF promoter were as follows: VEGF-1 $(-0.6 \mathrm{~kb})$ forward, 5'-GCAGACGGCAGTCACTAGG-3' and reverse, 5'-CACTCTCGAAGACGCTGCT-3'; VEGF-2 (-3.1 kb) forward, 5'-GAACATTTGGGAAATCTCTTGC-3' and reverse 5'-TGTGGCTCTGAAGGCAGTT-3'; VEGF-3 $(-3.2 \mathrm{~kb})$ forward, 5'-GCCTTGGCCCAATCACTAC-3' and reverse, 5'-GTGGAGAAAAGTGCGAAAGG-3'.

Lentiviral packaging. The cDNA of human transcription factor KLF4 (GenBank: BC030811/029923) was amplified by PCR, cloned into the vector pMD-18T (Takara, Dalian, China) 
and confirmed by DNA sequencing $(20,21)$. The KLF4 open reading frame (ORF) was then subcloned into the lentiviral vector pLenti-6.3 (Invitrogen). Viral packaging and infection were performed according to the standard protocols as recommended by the manufacturer (Invitrogen).

Western blotting. Total protein was extracted from the cells using lysis buffer containing $20 \mathrm{mM}$ Tris- $\mathrm{HCl}(\mathrm{pH} 7.4)$, $150 \mathrm{mM} \mathrm{NaCl}, 5 \mathrm{mM}$ EDTA, $1 \%$ Triton X-100, 1\% DTT and $1 \%$ protease inhibitor cocktail (Roche). Equal amounts of protein extracts $(40 \mu \mathrm{g})$ were separated by $10 \%$ sodium dodecyl sulfate-polyacrylamide gel electrophoresis (SDS-PAGE) and transferred onto a polyvinylidene fluoride (PVDF) membrane. Membranes were blocked with $5 \% \mathrm{w} / \mathrm{v}$ non-fat dry milk dissolved in Tris-buffered saline plus Tween-20 (TBS-T; 0.1\% Tween-20; $\mathrm{pH} 8.3$ ) at room temperature for $1 \mathrm{~h}$, then incubated with primary antibodies at $4^{\circ} \mathrm{C}$ overnight. The primary antibodies used were rabbit anti-KLF4 and anti- $\beta$-actin (Sigma). After washing with TBS-T, the membranes were incubated with horseradish peroxidase (HRP)-labeled secondary antibodies (Sigma) for 30-45 min at room temperature. Immunobands were visualized using enhanced chemiluminescence (ECL) kit (GE Healthcare, Waukesha, WI, USA) according to the manufacturer's instructions and exposed to X-ray film. $\beta$-actin was used as a loading control.

Statistical analysis. All statistical analyses were performed using the GraphPad Prism software, version 4 (GraphPad Software, San Diego CA, USA). Data are expressed as mean \pm SEM. Comparison of the means was performed using two-sided Student's t-test and a $\mathrm{P}<0.05$ value was considered to indicate a statistically significant result.

\section{Results}

Downregulation of miR-7 promotes the angiogenesis of HUVECs and induces the expression of VEGF and KLF4. We assessed the role of miR-7 in the angiogenesis of HUVECs by first transfecting HUVECs with a miR-7 inhibitor (miR-7 ASO). As shown in Fig. 1A, transient transfection of miR-7 ASO significantly decreased the miR-7 expression compared with that of the control. The tube formation assay was performed to evaluate the effect of miR-7 on the angiogenic potential of HUVECs. Knockdown of miR-7 significantly induced the tube formation of HUVECs (Fig. 1B and C) suggesting that miR-7 inhibition resulted in enhanced angiogenesis in the HUVECs.

VEGF plays a key role in endothelial cell migration and proliferation, and angiogenesis. We consequently assessed the VEGF expression following miR-7 inhibition. qRT-PCR analyses showed that VEGF mRNA levels were significantly increased in the HUVECs treated with miR-7 ASO (Fig. 1D). ELISA analyses further demonstrated significantly upregulated VEGF protein levels in the cell culture medium from the HUVECs treated with miR-7 ASO (Fig. 1E). Previous studies indicated that KLF4 is overexpressed in ECs and plays an important role in angiogenesis $(15,16)$. We further assessed the KLF4 expression following miR-7 inhibition in the HUVECs and found that the mRNA levels of KLF4 were significantly induced by miR-7 ASO (Fig. 1D). Similarly, the KLF4 protein level was markedly upregulated in the HUVECs treated with miR-7 ASO (Fig. 1F). These results demonstrated that downregulation of miR-7 promoted angiogenesis of HUVECs, accompanied by the upregulation of the expression of VEGF and KLF4.

miR-7 targets KLF4 in HUVECs. To explore the mechanism associated with the downregulation of miR-7 in angiogenesis, we searched for potential miR-7 target genes using miRNA target prediction tools, including TargetScan, PicTar and miRanda (22). We found that KLF4 is a potential miR-7 target. Previous studies have shown that KLF4 is a central regulator of sprouting angiogenesis via the Notch signaling pathway (20), and promoted epithelial cell transformation via targeting KLF4. These data suggested that miR-7 may inhibit angiogenesis by targeting KLF4 in HUVECs. To test this hypothesis, we analyzed the expression of KLF4 in HUVECs transfected with control or miR-7 mimics. Western blot analysis showed that miR-7 mimics decreased expression of KLF4 (Fig. 2A). Furthermore, qRT-PCR analysis revealed that the levels of KLF4 mRNA were obviously decreased in the HUVECs transfected with the miR-7 mimics (Fig. 2B). These results suggest that miR-7 may negatively regulate KLF4 expression by disrupting the stability of KLF4 mRNA.

To confirm that KLF4 is a direct target of miR-7, we constructed luciferase reporters with the KLF4 3'-UTR containing a miR-7 binding site (Fig. 2C). The constructs were transiently transfected into HUVECs along with miR-7 or NC mimics and the relative luciferase activity was measured at $48 \mathrm{~h}$. The relative luciferase activity of the construct with wild-type 3'-UTR was significantly repressed by $60 \%$ following miR-7 mimic transfection (Fig. 2D). However, site-directed mutagenesis of the miR-7 binding site within the 3'-UTR of KLF4 completely abolished this suppression (Fig. 2D), indicating that miR-7 directly binds to this site in HUVECs.

KLF4 activates VEGF transcription in HUVECs by directly interacting with the VEGF promoter. KLF4 is a transcription factor. The simultaneous promotion of the expression of KLF4 and VEGF in HUVECs after miR-7 inhibition suggests that KLF4 may promote VEGF transcription. Indeed, bioinformatic analysis identified the presence of multiple DNA-binding elements of the KLF4 transcription factor in the VEGF promoter (Fig. 3A). We then performed a ChIP and found that the KLF4 antibody specifically pulled down the VEGF promoter in the HUVECs (Fig. 3B). We next overexpressed KLF4 by infecting HUVECs with a lentivirus cloned with KLF4 (Lv-KLF4). Compared with the control lentivirus (Lv-Luc), infection of Lv-KLF4 significantly increased the mRNA (Fig. 3C) and protein level (Fig. 3D) of KLF4 in the HUVECs. As expected, ectopic overexpression of KLF4 significantly induced the VEGF mRNA levels in the HUVECs treated with miR-7 ASO (Fig. 3E), and the concentration of VEGF protein in the cell culture medium from HUVECs treated with miR-7 ASO (Fig. 3F). These results indicate that KLF4 playsed a direct role in the transcriptional regulation of VEGF.

KLF4 knockdown inhibits miR-7-knockdown-induced angiogenesis in HUVECs. Our observations showed that KLF4 is 
A

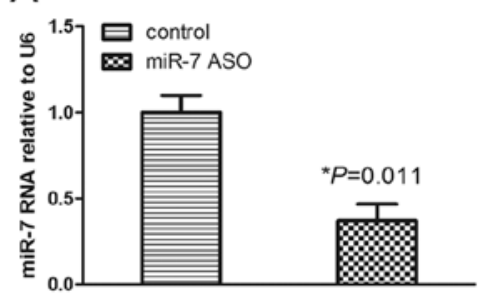

D

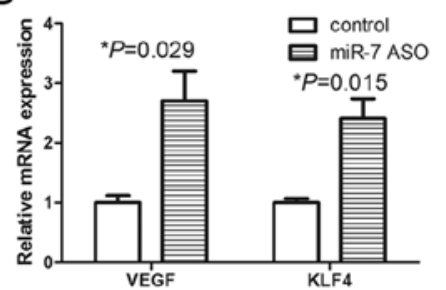

B

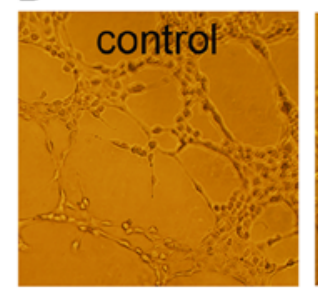

E

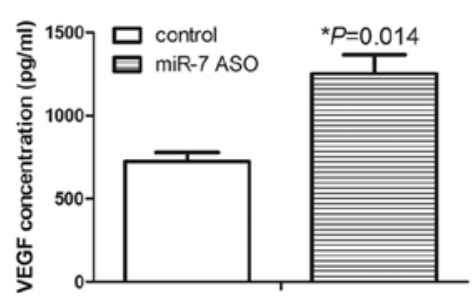

C
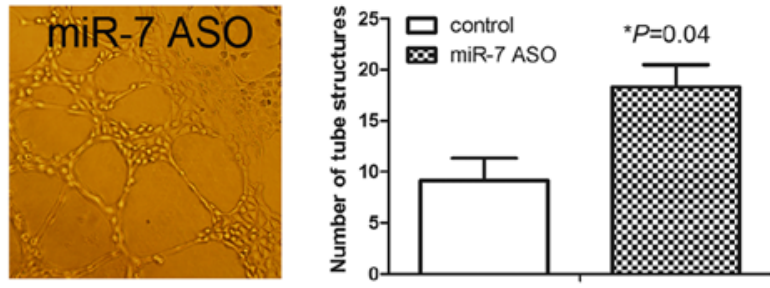

F

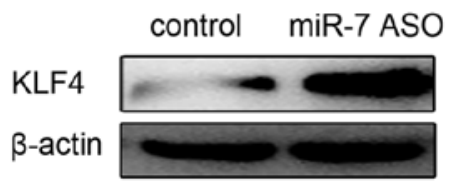

Figure 1. Transient transfection of miR-7 inhibitor promotes HUVEC tube formation and VEGF and KLF4 expression. (A) qRT-PCR analysis of miR-7 expression in HUVECs transfected with control or miR-7 ASO. (B) Images representative of HUVEC tube formation. (C) Quantification of HUVEC tube formation following miR-7 inhibition. (D) qRT-PCR analysis of VEGF and KLF4 after miR-7 knockdown. (E) VEGF protein in the cell culture medium from HUVECs treated with miR-7 ASO as assessed by ELISA. (F) Western blotting of KLF4 protein using $\beta$-actin as a loading control.

A

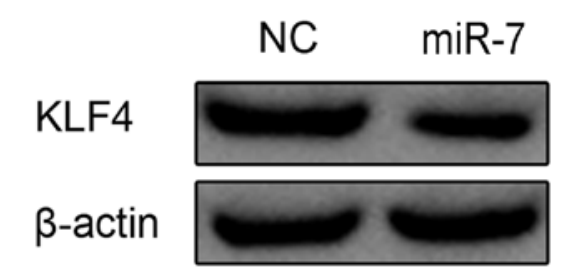

C

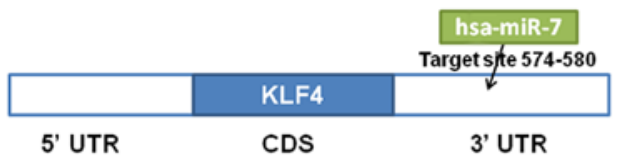

KLF4-UTR-wt 5'-ggaAaAucuauauUugucuUCCG -3' hsa-miR-7 3, UGUUGUUUUAGUGAU || | || |

KLF4-UTR-mut 5'- GGAAAAUCUAUAUUUCAGAAGGG -3'
B

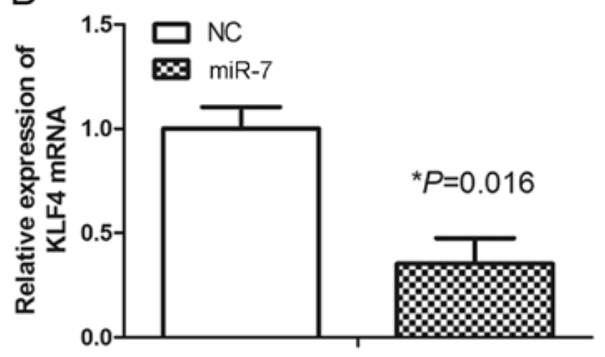

D

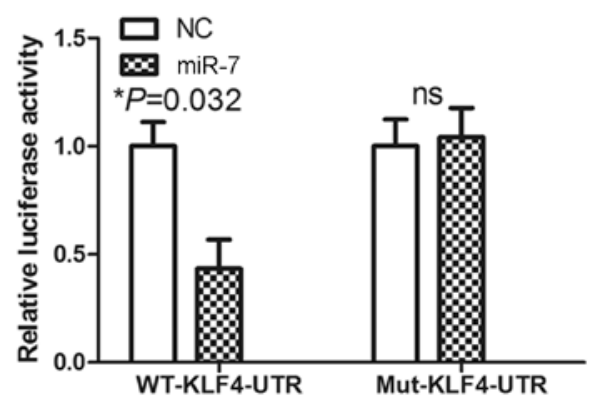

Figure 2. miR-7 targets KLF4 directly in HUVECs. (A) miR-7 overexpression suppressed endogenous KLF4 expression in the HUVECs. Western blotting of endogenous KLF4 protein expression in HUVECs infected with NC or miR-7 mimics; $\beta$-actin was used as an internal loading control. (B) Quantitative RT-PCR analysis of endogenous KLF4 mRNA expression in HUVECs transfected with NC or miR-7 mimics. (C) Schematic representation of the miR-7-binding sequence in the 3'-UTR of KLF4 mRNA. Mutations were generated in the miR-7-binding sequence of the KLF4 3'-UTR as indicated. (D) Analysis of luciferase activity in the HUVECs $48 \mathrm{~h}$ after co-transfection with the control Renilla luciferase expression construct pRL-TK and a Firefly luciferase reporter plasmid containing either the wild-type or mutant KLF4 3'-UTR (indicated as WT or MUT on the axis, respectively). Firefly luciferase activity in each sample was normalized to Renilla activity, and expressed relative to the normalized luciferase activity of control cells. ns, not significant.

a functional target of miR-7 and transcriptional activator of VEGF, which promotes angiogenesis, suggesting that knockdown of KLF4 may attenuate angiogenesis induced by miR-7 inhibition. We tested this hypothesis by transfecting exogenous KLF4 short hairpin RNA into the miR-7-transfected HUVECs using a previously described transient transfection system (11). KLF4 silencing significantly abolished the induction of VEGF
mRNA in the HUVECs treated with miR-7 ASO (Fig. 4A) and the concentration of VEGF protein in the cell culture medium from HUVECs treated with miR-7 ASO (Fig. 4B). Consistent with the key function of VEGF in angiogenesis, KLF4 knockdown significantly prevented the induction of angiogenesis by the miR-7 inhibitor in the HUVECs (Fig. 4C and D). Taken together, the results indicate that KLF4 knockdown abrogates 
A

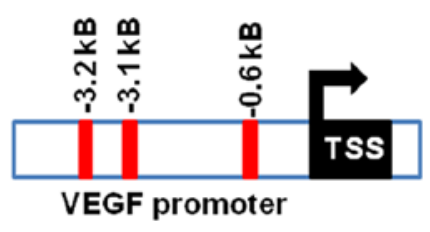

D

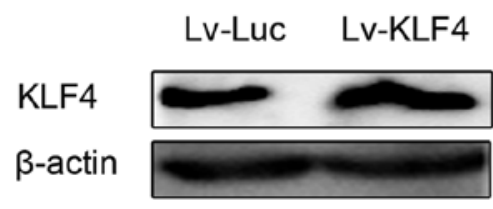

B

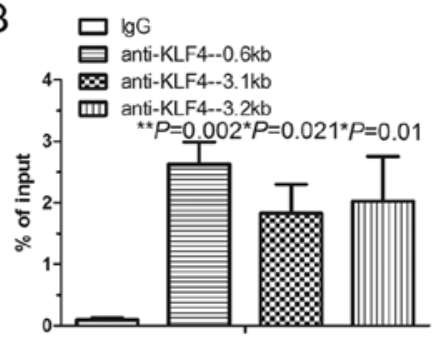

E

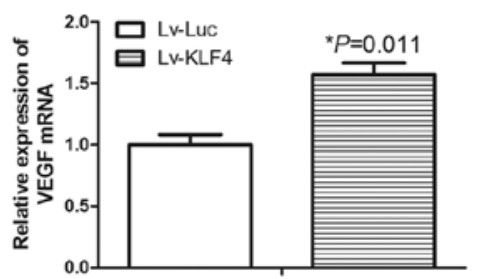

C

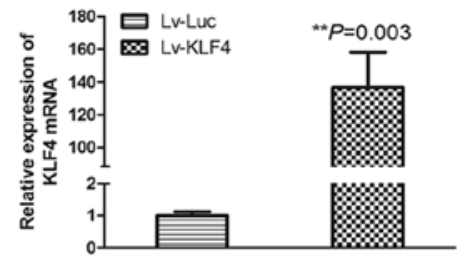

$\mathrm{F}$

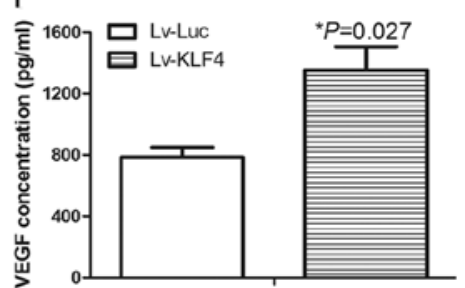

Figure 3. KLF4 binds with the VEGF promoter to trigger VEGF transcription. (A) Bioinformatic analysis showing the VEGF promoter with 3 putative KLF4-binding sites. (B) ChIP assay. HUVECs were grown and treated with miR-7 and subjected to ChIP and qPCR analysis of the KLF4-binding sites at the VEGF promoter. The relative enrichment data were normalized to IgG as control. The assays in B and C were performed in triplicate. (C) qRT-PCR analysis of KLF4 mRNA after KLF4 overexpression in the lentivirus-transfected HUVECs. (D) Western blotting of KLF4 after overexpression of KLF4 in the lentivirus-transfected HUVECs. (E) qRT-PCR analysis of VEGF mRNA after overexpression of KLF4 in the lentivirus-transfected HUVECs. (F) ELISA of VEGF in the cell culture after overexpression of KLF4 in the lentivirus-transfected HUVECs.

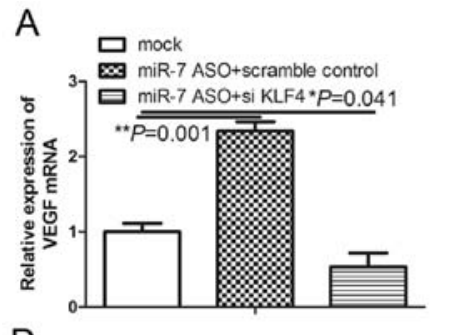

D

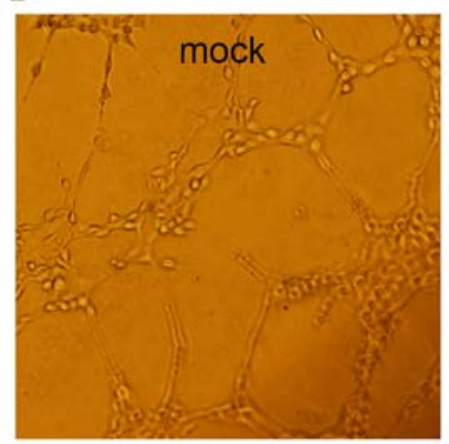

B
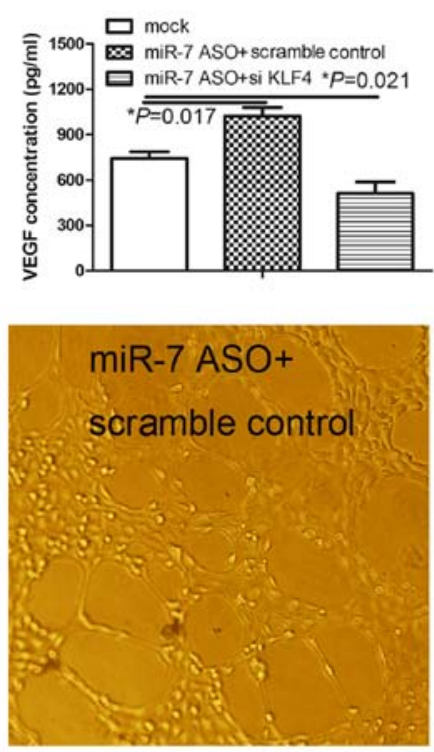

C
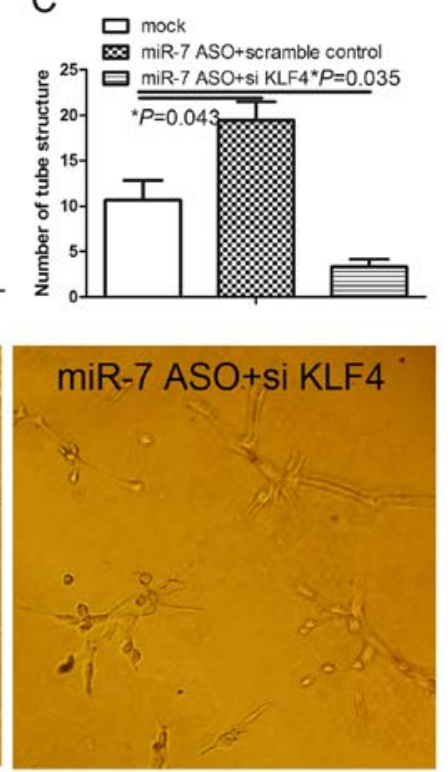

Figure 4. KLF4 mediates miR-7-knockdown-induced angiogenesis in HUVECs. (A) qRT-PCR analysis of VEGF mRNA following KLF4 knockdown using a KLF4-targeting shRNA in HUVECs transfected with the miR-7 inhibitor. (B) ELISA of VEGF in the cell culture following KLF4 knockdown using a KLF4-targeting shRNA in HUVECs transfected with the miR-7 inhibitor. (C) Quantification of HUVEC tube formation following knockdown of KLF4 expression with a KLF4-targeting shRNA in HUVECs transfected with the miR-7 inhibitor. (D) Images representing tube formation of HUVECs following KLF4 knockdown with a KLF4-targeting shRNA in HUVECs transfected with the miR-7 inhibitor.

miR-7-knockdown-induced angiogenesis, suggesting that KLF4 is a functional mediator of miR-7 in the regulation of angiogenesis in HUVECs.

\section{Discussion}

In the present study, we demonstrated that miR-7 downregulation promoted the angiogenesis of HUVECs, accompanied by the induction of VEGF and KLF4 expression. Furthermore, we found that miR-7 directly targeted KLF4 and KLF4 promoted the gene transcription of VEGF by directly interacting with the VEGF promoter in the HUVECs. Consequently, KLF4 silencing inhibited miR-7-knockdown-induced angiogenesis in HUVECs. These findings indicate that the miR-7-KLF4-VEGF signaling axis plays an important role in the regulation of angiogenesis in HUVECs, suggesting that the miR-7-KLF4 
signaling pathway is a potential target for development of antiangiogenic therapeutics in vascular diseases as well as solid tumors.

Growing evidence has shown that aberrant expression of miRNAs is directly associated with the development and progression of several types of cancers (23). Deregulation of miR-7 has been found in cervical (24), breast $(25,26)$, colorectal (27) and lung cancer (28). It was recently reported that miR-7 overexpression inhibited tumor angiogenesis and the growth of murine xenograft glioblastoma (12). Consistent with the present study, we found that downregulation of miR-7 led to enhanced tube formation in HUVECs. Furthermore, our results showed that the miR-7 inhibitor increased VEGF by targeting KLF4. These data suggest that miR-7 is an angiogenesis inhibitor and a potential agent for the development of anti-angiogenic therapeutics.

VEGF is the most potent pro-angiogenesis factor and plays a crucial role in tumor angiogenesis and metastatic spread of cancer cells. Thus, targeting VEGF is a very promising strategy for cancer treatment, with numerous anti-VEGF small molecules in preclinical and clinical trials (29). However, the precise molecular mechanism of VEGF regulation in HUVECs remains to be determined. Zheng et al found that overexpression of KLF4 in ECs significantly impaired tube formation (30). It was reported that defective angiogenesis led to reduced tumor growth independent of endothelial cell proliferation (31). KLF4 is an upstream regulator of Notch signaling and sustained expression of KLF4 leads to defective angiogenesis, resulting in attenuation of tumor growth (20). By contrast, Hale et al found that sustained expression of KLF4 promoted sprouting angiogenesis in the HUVECs (20). Consistent with the present study, we found that KLF4 positively regulated the expression of VEGF in HUVECs. Therefore, further animal models are needed to clarify the functions of KLF4 in the regulation of angiogenesis in solid tumors.

It is well documented that a single miRNA can post-transcriptionally suppress multiple targets. It is, therefore, quite possible that miR-7 may also target other angiogenesis-promoting genes in addition to KLF4 simultaneously, to promote angiogenesis. For example, a recent study indicated that miR-7-5p was frequently downregulated in glioblastoma microvasculature, and miR-7-5p overexpression in HUVECs inhibited vascular endothelial cell proliferation by targeting RAF1 (32). Babae et al found that miR-7 is a novel anti-angiogenic therapeutic miRNA that was systemically delivered into both ECs and tumor cells, suggesting a potential therapeutic role for miR-7 as a novel antitumor agent (12). Similarly, we observed that miR-7 knockdown promoted HUVEC angiogenesis, accompanied by increased levels of both VEGF and KLF4, explaining the promotion of angiogenesis by miR-7 inhibitors. It should be noted that VEGF is not a direct target gene of miR-7. Our findings suggest that KLF4 is a mediator between miR-7 and VEGF in the regulation of angiogenesis.

In summary, we found that KLF4 is a direct target of miR-7 and transcription activator of VEGF in HUVECs, revealing an additional mechanism underlying the inhibition of miR-7 in angiogenesis. VEGF plays a key role in endothelial cell migration, proliferation and angiogenesis $(33,34)$. Our findings suggest that miR-7 downregulation may contribute to sustained angiogenesis in cancer.

\section{Acknowledgements}

The present study was supported by the Science and Technology Development Program of Shaanxi Province of China (2013KJXX-91).

\section{References}

1. Nakatsu MN, Sainson RC, Aoto JN, Taylor KL, Aitkenhead M, Pérez-del-Pulgar S, Carpenter PM and Hughes CC: Angiogenic sprouting and capillary lumen formation modeled by human umbilical vein endothelial cells (HUVEC) in fibrin gels: The role of fibroblasts and Angiopoietin-1. Microvasc Res 66: 102-112, 2003.

2. Conway EM, Collen D and Carmeliet P: Molecular mechanisms of blood vessel growth. Cardiovasc Res 49: 507-521, 2001.

3. Yu X, Zhao R, Lin S, Bai X, Zhang L, Yuan S and Sun L: CXCL16 induces angiogenesis in autocrine signaling pathway involving hypoxia-inducible factor $1 \alpha$ in human umbilical vein endothelial cells. Oncol Rep 35: 1557-1565, 2016.

4. Wu Z, Cai X, Huang C, Xu J and Liu A: miR-497 suppresses angiogenesis in breast carcinoma by targeting HIF-1 $\alpha$. Oncol Rep 35: 1696-1702, 2016

5. Yoshikawa N, Shimizu N, Ojima H, Kobayashi H, Hosono O and Tanaka H: Down-regulation of hypoxia-inducible factor-1 alpha and vascular endothelial growth factor by HEXIM1 attenuates myocardial angiogenesis in hypoxic mice. Biochem Biophys Res Commun 453: 600-605, 2014.

6. Dong W, Li B, Wang J, Song Y, Zhang Z, Fu C and Zhang P: Diagnostic and predictive significance of serum microRNA-7 in esophageal squamous cell carcinoma. Oncol Rep 35: 1449-1456, 2016.

7. Zhang H, Qu Y, Duan J, Deng T, Liu R, Zhang L, Bai M, Li J, Zhou L, Ning T, et al: Integrated analysis of the miRNA, gene and pathway regulatory network in gastric cancer. Oncol Rep 35: 1135-1146, 2016.

8. Chakrabarti M and Ray SK: Antitumor activities of luteolin and silibinin in glioblastoma cells: Overexpression of miR-7-1-3p augmented luteolin and silibinin to inhibit autophagy and induce apoptosis in glioblastoma in vivo. Apoptosis 21: 312-328, 2016.

9. Zhao JG, Men WF and Tang J: MicroRNA-7 enhances cytotoxicity induced by gefitinib in non-small cell lung cancer via inhibiting the EGFR and IGF1R signalling pathways. Contemp Oncol 19: 201-206, 2015.

10. Zhang N, Li X, Wu CW, Dong Y, Cai M, Mok MT, Wang H, Chen J, Ng SS, Chen M, et al: microRNA-7 is a novel inhibitor of YY1 contributing to colorectal tumorigenesis. Oncogene 32: 5078-5088, 2013.

11. Zhang X, Hu S, Zhang X, Wang L, Zhang X, Yan B, Zhao J, Yang A and Zhang R: MicroRNA-7 arrests cell cycle in G1 phase by directly targeting CCNE1 in human hepatocellular carcinoma cells. Biochem Biophys Res Commun 443: 1078-1084, 2014.

12. Babae N, Bourajjaj M, Liu Y, Van Beijnum JR, Cerisoli F, Scaria PV, Verheul M, Van Berkel MP, Pieters EH, Van Haastert RJ, et al: Systemic miRNA-7 delivery inhibits tumor angiogenesis and growth in murine xenograft glioblastoma. Oncotarget 5: 6687-6700, 2014.

13. Garrett-Sinha LA, Eberspaecher H, Seldin MF and de Crombrugghe B: A gene for a novel zinc-finger protein expressed in differentiated epithelial cells and transiently in certain mesenchymal cells. J Biol Chem 271: 31384-31390, 1996.

14. Takahashi K and Yamanaka S: Induction of pluripotent stem cells from mouse embryonic and adult fibroblast cultures by defined factors. Cell 126: 663-676, 2006.

15. Atkins GB and Jain MK: Role of Krüppel-like transcription factors in endothelial biology. Circ Res 100: 1686-1695, 2007.

16. Mun GI and Boo YC: A regulatory role of Kruppel-like factor 4 in endothelial argininosuccinate synthetase 1 expression in response to laminar shear stress. Biochem Biophys Res Commun 420: 450-455, 2012.

17. Ohnesorge N, Viemann D, Schmidt N, Czymai T, Spiering D, Schmolke M, Ludwig S, Roth J, Goebeler M and Schmidt M: Erk5 activation elicits a vasoprotective endothelial phenotype via induction of Kruppel-like factor 4 (KLF4). J Biol Chem 285: 26199-26210, 2010. 
18. Zhang L, Zhou M, Qin G, Weintraub NL and Tang Y: MiR-92a regulates viability and angiogenesis of endothelial cells under oxidative stress. Biochem Biophys Res Commun 446: 952-958, 2014.

19. Cui Y, Xiao Z, Chen T, Wei J, Chen L, Liu L, Chen B, Wang X, Li X and Dai J: The miR-7 identified from collagen biomaterialbased three-dimensional cultured cells regulates neural stem cell differentiation. Stem Cells Dev 23: 393-405, 2014

20. Hale AT, Tian H, Anih E, Recio FO III, Shatat MA, Johnson T, Liao X, Ramirez-Bergeron DL, Proweller A, Ishikawa M, et al: Endothelial Kruppel-like factor 4 regulates angiogenesis and the Notch signaling pathway. J Biol Chem 289: 12016-12028, 2014.

21. Pan C, Lu B, Chen H and Bishop CE: Reprogramming human fibroblasts using HIV-1 TAT recombinant proteins OCT4, SOX2, KLF4 and c-MYC. Mol Biol Rep 37: 2117-2124, 2010.

22. Thomas M, Lieberman J, Lal A and Lal A: Desperately seeking microRNA targets. Nat Struct Mol Biol 17: 1169-1174, 2010.

23. Wang GJ, Liu GH, Ye YW, Fu Y and Zhang XF: The role of microRNA-1274a in the tumorigenesis of gastric cancer: Accelerating cancer cell proliferation and migration via directly targeting FOXO4. Biochem Biophys Res Commun 459: 629-635, 2015.

24. Hao Z, Yang J, Wang C, Li Y, Zhang Y, Dong X, Zhou L, Liu J, Zhang Y and Qian J: MicroRNA-7 inhibits metastasis and invasion through targeting focal adhesion kinase in cervical cancer. Int J Clin Exp Med 8: 480-487, 2015.

25. Huynh FC and Jones FE: MicroRNA-7 inhibits multiple oncogenic pathways to suppress HER $2 \Delta 16$ mediated breast tumorigenesis and reverse trastuzumab resistance. PLoS One 9: e114419, 2014

26. Shi Y, Luo X, Li P, Tan J, Wang X, Xiang T and Ren G: miR-7-5p suppresses cell proliferation and induces apoptosis of breast cancer cells mainly by targeting REG $\gamma$. Cancer Lett 358: 27-36, 2015.

27. Suto T, Yokobori T, Yajima R, Morita H, Fujii T, Yamaguchi S, Altan B, Tsutsumi S, Asao T and Kuwano H: MicroRNA-7 expression in colorectal cancer is associated with poor prognosis and regulates cetuximab sensitivity via EGFR regulation. Carcinogenesis 36: 338-345, 2015.
28. Li J, Zheng Y, Sun G and Xiong S: Restoration of miR-7 expression suppresses the growth of Lewis lung cancer cells by modulating epidermal growth factor receptor signaling. Oncol Rep 32: 2511-2516, 2014.

29. D'Alessandris QG, Martini M, Cenci T, Capo G, Ricci-Vitiani L, Larocca LM and Pallini R: VEGF isoforms as outcome biomarker for anti-angiogenic therapy in recurrent glioblastoma. Neurology 84: 1906-1908, 2015.

30. Zheng X, Li A, Zhao L, Zhou T, Shen Q, Cui Q and Qin X: Key role of microRNA-15a in the KLF4 suppressions of proliferation and angiogenesis in endothelial and vascular smooth muscle cells. Biochem Biophys Res Commun 437: 625-631, 2013.

31. Ray A, Alalem M and Ray BK: Loss of epigenetic Kruppel-like factor 4 histone deacetylase (KLF-4-HDAC)-mediated transcriptional suppression is crucial in increasing vascular endothelial growth factor (VEGF) expression in breast cancer. J Biol Chem 288: 27232-27242, 2013.

32. Liu Z, Liu Y, Li L, Xu Z, Bi B, Wang Y and Li JY: MiR-7-5p is frequently downregulated in glioblastoma microvasculature and inhibits vascular endothelial cell proliferation by targeting RAF1. Tumour Biol 35: 10177-10184, 2014.

33. Koch AE, Harlow LA, Haines GK, Amento EP, Unemori EN, Wong WL, Pope RM and Ferrara N: Vascular endothelial growth factor. A cytokine modulating endothelial function in rheumatoid arthritis. J Immunol 152: 4149-4156, 1994.

34. Melter M, Reinders ME, Sho M, Pal S, Geehan C, Denton MD, Mukhopadhyay D and Briscoe DM: Ligation of CD40 induces the expression of vascular endothelial growth factor by endothelial cells and monocytes and promotes angiogenesis in vivo. Blood 96: 3801-3808, 2000 